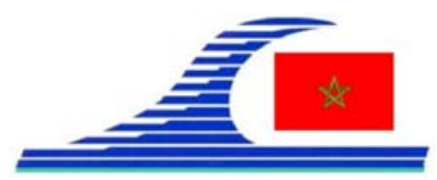

Conférence Méditerranéenne Côtière et Maritime EDITION 2, TANGER, MAROC (2011)

Coastal and Maritime Mediterranean Conference

Disponible en ligne - http://www.paralia.fr-Available online

\title{
Origines de la salinité des eaux dans la zone littorale Annaba Guerbes (Algérie)
}

\author{
Larbi DJABRI ${ }^{1}$, Noureddine GUEZGOUZ ${ }^{1}$, Saad BOUHSINA ${ }^{2}$, \\ Samia HADJ- SAID ${ }^{3}$, Azzedine HANI ${ }^{1}$, \\ Antonio PULIDO BOSCH ${ }^{4}$, Yacine DJABRI ${ }^{5}$
}

\author{
1. Université Badji Mokhtar, Annaba, Algérie.Djabri_larbi@yahoo.fr \\ 2. Université du littoral, Dunkerque, France. \\ 3. Université de Ouargla. Algérie.samia_h2001@yahoo.fr \\ 4. Université d’Almeria, Département d'Hydrogéologie.04120 Almeria, Espagne. \\ 5. CHU, IBN Rochd Service de Gynécologie Obstétrique. Annaba, Algérie.
}

\section{Résumé :}

La nappe côtière de Guerbes est contenue dans les sables dunaires, ces formations très perméables facilitent les échanges entre la nappe et son environnement. Les nombreux marécages présents peuvent influencer le chimisme des eaux. Les eaux des puits présentent une certaine minéralisation, caractérisée par des valeurs importantes de la conductivité électrique de l'ordre de $4000 \mu \mathrm{S} \mathrm{cm}^{-1}$.

La zone d'étude est caractérisée par une pluviosité importante de l'ordre de $1000 \mathrm{~mm} \mathrm{an}^{-1}$, cet apport en eau douce n'atténue pas beaucoup la salinité des eaux dont la valeur reste considérablement influencée par la mer. Les échanges directs ou indirects s'effectuant entre la nappe et la mer semblent influencer cette salinité. Par le biais de ce travail, nous allons essayer de mettre en évidence cette relation de cause à effet entre la salinité des eaux de la nappe et la mer.

\section{Mots-clés :}

Eaux souterraines - Salinité - Intrusion marine - Algérie

\section{Introduction}

Les travaux ayant traité de la salinité des eaux, sont nombreux et diversifiés. Ainsi au Maroc, HSISSOU et al. (1997) ont mis en évidence l'influence du biseau salé et des faciès évaporitiques sur la salinité des eaux de la nappe côtière d’Agadir. En Algérie, les travaux effectués par DJABRI (1996) et HANI (2003), mettent en évidence la salinité des eaux dans deux aquifères à climat et géologie contrastés: nappe alluviale de Tébessa (semi-aride) et nappe superficielle d'Annaba-Bouchegouf-Guelma. HADJSAID (2007) a combiné les données géophysiques et chimiques pour expliquer la qualité des eaux de la région de Guerbes. En Espagne PULIDO-LEBOEUF et al. (2003) ont traité de l’origine de la salinité des eaux dans des aquifères. Par le présent travail nous allons essayer d'expliquer les mécanismes d'acquisition de la salinité ce qui nous 
La connaissance de la Mer :

un vecteur du développement durable en Méditerranée

permettra l'élaboration d'un modèle mathématique hydrodispersif qui tiendra compte de tous les paramètres régissant la salinité.

\section{Site étudié}

La région de Guerbes fait partie de la côte orientale algérienne. Elle est située entre les monts de Filfila et Safia au sud-ouest, les massifs de Cap de Fer et l'Edough au nord-est et ceux de Bou Maiza et Berrahal au sud-est (figure 1). Le climat de la zone d'étude est de type méditerranéen, la pluviométrie est de l'ordre de $750 \mathrm{~mm}^{-1}{ }^{-1} \mathrm{et}$ la température moyenne annuelle est de $18^{\circ} \mathrm{C}$. La démographique et l'agriculture génèrent une demande en eau importante, conduisant à des pompages intensifs entrainant une surexploitation de la nappe et par conséquent une dégradation de la qualité des eaux (augmentation de la minéralisation).

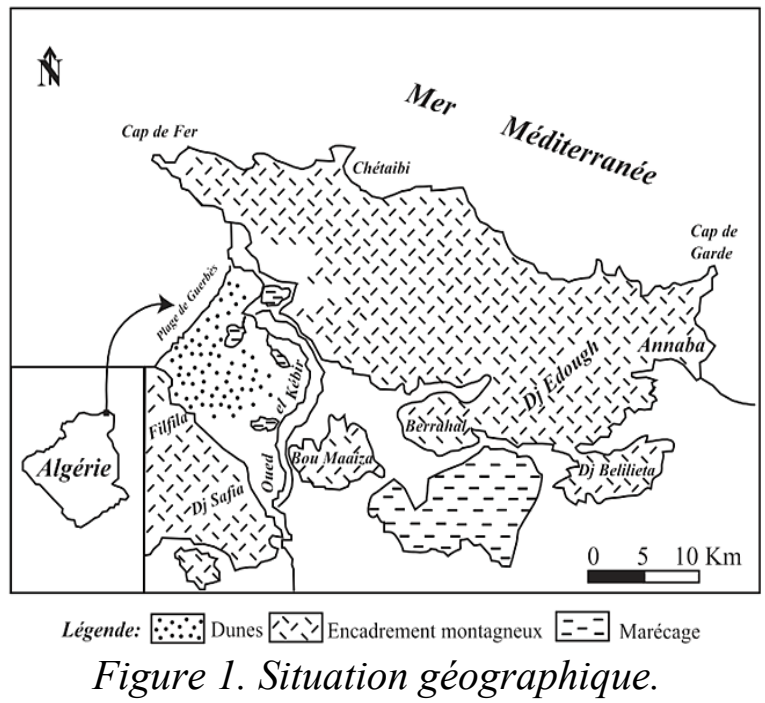

\section{Matériels et méthodes}

On a utilisé les données hydrogéologiques et hydrochimiques :

- L'outil hydrogéologique; la description de l'aquifère. Elaborée à partir des données de forages et de puits. Les caractéristiques hydrodynamiques $\mathrm{T}$ (Transmissivité), $\mathrm{K}$ (conductivité hydraulique), S (coefficient d'emmagasinement) et les cartes piézométriques, donnent un aperçu sur la nature de la nappe.

- Les analyses d'eau donnent un aperçu sur la qualité des eaux. Les paramètres physiques ont été mesurés sur le terrain à l'aide d'un appareil multi paramètres WTW multiline P4.

- L'analyse chimique de l'eau réalisée par la méthode de spectrophotométrie d'absorption moléculaire. 


\section{Résultats et discussions}

La nappe libre de Guerbes est contenue dans des formations plio-quaternaires, constituées de sables dunaires marins. Son substratum partiellement érodé est formé par les argiles numidiennes. La Transmissivité est comprise entre 0,3 et $0,610^{-3} \mathrm{~m}^{2} \mathrm{~s}^{-1}$ au sud et de 2,8 $10^{-3} \mathrm{~m}^{2} \mathrm{~s}^{-1}$ au nord-ouest à Sidi Lakhdar. La carte piézométrique (figure 2), montre au nord et au sud-est une zone de dépression due à l'existence des marais. Au sud, apparait 'un dôme piézométrique constituant une zone de recharge de la nappe. Dans le secteur côtier, l'écoulement est dirigé du nord vers le sud, généré soit par un changement topographique soit par les pompages intensifs.

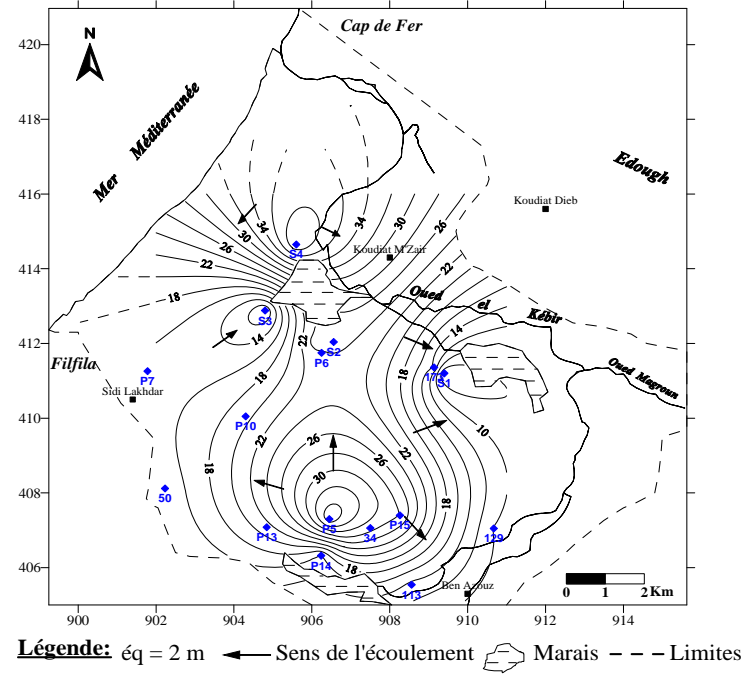

Figure 2. Carte piézométrique et puits mesurés.

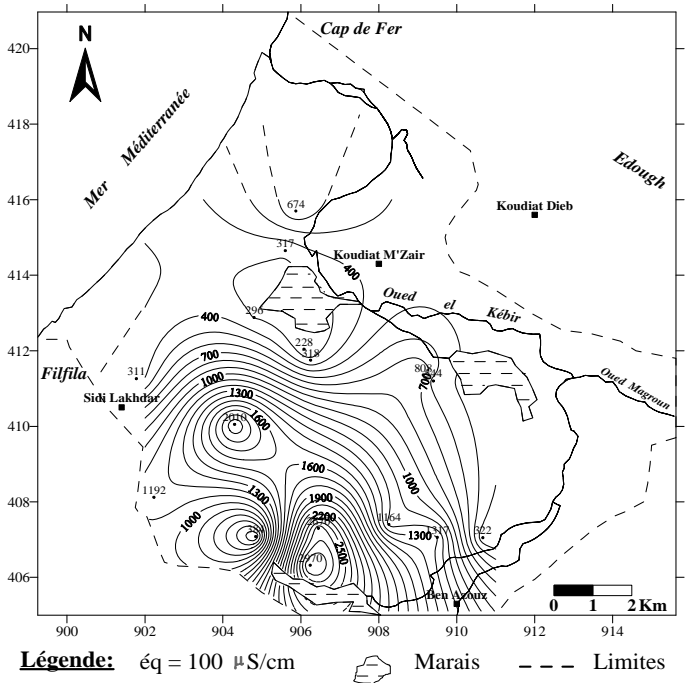

Figure 3. Carte des conductivités.

\subsection{Hydrochimie}

La carte des conductivités (figure 3), montre que les zones les plus chargées se localisent au centre et au sud de la zone d'étude. Par contre au niveau du secteur côtier les conductivités sont de l'ordre de $1000 \mu \mathrm{S} \mathrm{cm}{ }^{-1}$. Cette répartition est influencée par la piézométrie. Pour expliquer les origines de cette salinité, nous avons utilisé les rapports suivants : $\mathrm{Mg}^{2+} / \mathrm{Ca}^{2+}, \mathrm{SO}_{4}{ }^{2-} / \mathrm{Cl}^{-}$et $\mathrm{Cl} / \sigma$ ( $\sigma=$ conductivité). La combinaison des résultats nous permettra de déterminer l’impact de la mer sur la salinité des eaux.

\subsection{Application de la méthode des rapports}

La méthode des rapports reste la plus efficace et la plus simple à réaliser, elle se base sur des valeurs seuils. Les puits analysés sont situés à différentes distances de la mer, ce qui nous permet de constater l'influence marine sur la qualité des eaux. A partir des valeurs obtenues, on déterminera l'origine de la salinité. Ainsi les valeurs seuils, qui nous permettront de trancher quand à l'origine de la salinité sont : $\mathrm{Mg}^{2+} / \mathrm{Ca}^{2+}=4,5$; 
La connaissance de la Mer :

un vecteur du développement durable en Méditerranée

$\mathrm{SO}_{4}{ }^{2-} / \mathrm{Cl}^{-}=0,1 ; \mathrm{Cl}^{-} / \sigma=0,307$. Ainsi, si $\mathrm{Cl}^{-} / \sigma<0,307$ les chlorures ont une origine marine, mais si $\mathrm{Cl}^{\top} / \sigma>0,307$ les chlorures n'ont pas une origine marine. En utilisant les valeurs seuils et en observant le tableau 1, nous pouvons déduire les remarques suivantes :

Pour le rapport $\mathrm{Mg}^{2+} / \mathrm{Ca}^{2+}$ : seul deux points indiquent une salinité influencée par la mer, c'est le cas des points P5 et P6, d'autres points présentent une forte valeur du rapport oscillant entre 3,5 et 3,9 ce qui semble indiquer un mélange des eaux traduisant diverses origines de la salinité. Le reste des points montre une salinité faible, provoquée par la dilution des eaux lors du contact eau-roche. Dans le cas du rapport $\mathrm{SO}_{4}{ }^{2-} / \mathrm{Cl}^{-}$: $70 \%$, des échantillons indiquent une origine marine des chlorures, ce qui implique une salinité marine des eaux. Concernant le rapport $\mathrm{Cl}^{-} / \sigma$ : 82\% des échantillons, montrent un rapport inférieur 0,307 ce qui implique une origine marine des chlorures.

Tableau 1. Valeurs des rapports caractéristiques par rapport à la distance à la mer.

\begin{tabular}{|c|c|c|c|c|}
\hline Puits & $\begin{array}{l}\text { Distance à la mer } \\
(\mathrm{km})\end{array}$ & $\begin{array}{l}\mathrm{Mg}^{2+} / \mathrm{Ca}^{2+} \\
\text { Valeur seuil }=4,5\end{array}$ & $\begin{array}{l}\mathrm{SO}_{4}{ }^{2-} / \mathrm{Cl} \\
\text { Valeur Seuil = 0,1 }\end{array}$ & $\begin{array}{l}\mathrm{Cr} / \sigma \\
\text { Valeur Seuil }=0,307\end{array}$ \\
\hline$S 1$ & 2,7 & 3 & 0,05 & 0,32 \\
\hline$P 7$ & 2,75 & 3,4 & 0,15 & 0,29 \\
\hline$S 2$ & 3,33 & 1,9 & 0,06 & 0,28 \\
\hline 50 & 5,21 & 3,9 & 0,2 & 0,18 \\
\hline P6 & 5,7 & 6,2 & 0,2 & 0,27 \\
\hline$S 3$ & 6 & 3,8 & 0,1 & 0,36 \\
\hline$P 10$ & 6,45 & 3,7 & 0,37 & 0,13 \\
\hline$P 13$ & 8 & 2,5 & 0,01 & 0,21 \\
\hline S4 & 8,1 & 2,5 & 0,17 & 0,26 \\
\hline 173 & 8,19 & 2,1 & 0,34 & 0,2 \\
\hline P5 & 9 & 4,7 & 0,6 & 0,11 \\
\hline P14 & 9,6 & 2,9 & 0,35 & 0,09 \\
\hline 34 & 9,68 & 1,9 & 0,3 & 0,13 \\
\hline P15 & 10,3 & 2 & 0,12 & 0,18 \\
\hline 113 & 11,65 & 3,5 & 0,05 & 0,2 \\
\hline 129 & 12,7 & 3,8 & 0,06 & 0,34 \\
\hline
\end{tabular}

\section{Conclusion}

Le présent travail montre que la salinité des eaux peut avoir diverses origines, en effet les résultats obtenus à partir de l'utilisation des différents rapports, ne montrent pas une convergence franche. Ainsi nous pouvons déduire que l'influence marine est présente, cependant cette influence s'atténue au fur et à mesure qu’on s’éloigne du littoral. 


\section{Références bibliographiques}

DJABRI L. (1996). Mécanisme de la pollution et vulnérabilité des eaux de la Seybouse. Origines géologiques, industrielles, agricoles et urbaine. Thèse d'Etat, Université de Annaba, $176 \mathrm{p}$.

HADJ-SAID S. (2007). Contribution à l'étude hydrogéologique d'un aquifère en zone côtière : cas de la nappe de Guerbes. Thèse, Université de Annaba, 188 p.

HSISSOU Y., MUDRY J., MANIA J., BOUCHAOU L., CHAUVE P. (1997). Dynamique et salinité de la nappe côtière d'Agadir (Maroc): Influence du biseau salé et des faciès évaporitiques. Hydrochemistry, IAHS Publ., n² 244, pp 73-82.

HANI A. (2003). Analyse méthodologique de la structure et des processus anthropiques: Application aux ressources en eau d'un bassin côtier méditerranéen. Thèse de Doctorat d'Etat, Université de Annaba, 209 p.

PULIDO-LEBOEUF P., PULIDO-BOSCH A., CALVACHE M.L., VALLEJOS A., ANDREU J.M. (2003). Strontium, $\mathrm{SO}_{4}{ }^{2-} / \mathrm{Cl}^{-}$and $\mathrm{Mg}^{2+} / \mathrm{Ca}^{2+}$ ratios as tracers for the evolution of seawater into coastal aquifers: the example of Castell de Ferro aquifer (SE Spain). Comptes Rendus Geoscience, Volume 335(14), pp 1039-1048. doi:10.1016/j.crte.2003.08.004 
La connaissance de la Mer :

un vecteur du développement durable en Méditerranée 